\title{
Gestational diabetes mellitus and long-term consequences for mother and offspring: a view from Denmark
}

\author{
Peter Damm ${ }^{1,2}$ • Azadeh Houshmand-Oeregaard ${ }^{1,2,3}$ - Louise Kelstrup ${ }^{1,2,3}$. \\ Jeannet Lauenborg ${ }^{2,4}$. Elisabeth R. Mathiesen ${ }^{1,2}$. Tine D. Clausen ${ }^{2,5}$
}

Received: 18 March 2016/Accepted: 30 March 2016/Published online: 12 May 2016

(C) Springer-Verlag Berlin Heidelberg 2016

\begin{abstract}
Gestational diabetes mellitus (GDM) is defined as glucose intolerance of varying severity and is present in about $2-6 \%$ of all pregnancies in Europe, making it one of the most common pregnancy disorders. Aside from the short-term maternal, fetal and neonatal consequences associated with GDM, there are long-term consequences for both mother and child. Although maternal glucose tolerance often normalises shortly after pregnancy, women with GDM have a substantially increased risk of developing type 2 diabetes later in life. Studies have reported that women are more than seven times as likely to develop diabetes after GDM, and that approximately $50 \%$ of mothers with GDM will develop diabetes within 10 years, making GDM one of the strongest predictors of type 2 diabetes. In women with previous GDM, development of type 2 diabetes can be prevented or delayed by lifestyle intervention and/or medical treatment. Systematic follow-up programmes would be ideal to prevent progression of GDM to diabetes, but such programmes are unfortunately lacking in the routine clinical set-up in most countries. Studies have found that the risks of obesity, the metabolic
\end{abstract}

Peter Damm

pdamm@dadlnet.dk

1 Center for Pregnant Women with Diabetes, Departments of Endocrinology and Obstetrics 4031, Rigshospitalet, Blegdamsvej 9, DK-2100 Copenhagen, Denmark

2 Institute of Clinical Medicine, Faculty of Health and Medical Sciences, University of Copenhagen, Copenhagen, Denmark

3 Diabetes and Metabolism, Department of Endocrinology, Rigshospitalet, Copenhagen, Denmark

4 Department of Obstetrics and Gynecology, Herlev Hospital, Herlev, Denmark

5 Department of Obstetrics and Gynecology, Nordsjællands Hospital, Hilleroed, Denmark syndrome, type 2 diabetes and impaired insulin sensitivity and secretion in offspring of mothers with GDM are two- to eightfold those in offspring of mothers without GDM. The underlying pathogenic mechanisms behind the abnormal metabolic risk profile in offspring are unknown, but epigenetic changes induced by exposure to maternal hyperglycaemia during fetal life are implicated. Animal studies indicate that treatment can prevent long-term metabolic complications in offspring, but this remains to be confirmed in humans. Thus, diabetes begets diabetes and it is likely that GDM plays a significant role in the global diabetes epidemic. This review summarises a presentation given at the 'Gestational diabetes: what's up?' symposium at the 2015 annual meeting of the EASD. It is accompanied by two other reviews on topics from this symposium (by Marja Vääräsmäki, DOI: 10.1007/s00125-016-3976-6, and by Cuilin Zhang and colleagues, DOI: 10.1007/s00125-016-3979-3) and an overview by the Session Chair, Kerstin Berntorp (DOI: 10.1007/s00125-016-3975-7).

Keywords Gestational diabetes · Long-term • Mother · Offspring $\cdot$ Review $\cdot$ Risk

Abbreviations
$\begin{array}{ll}\text { GDM } & \text { Gestational diabetes mellitus } \\ \text { O-BP } & \begin{array}{l}\text { Offspring of women from the } \\ \text { background population }\end{array} \\ \text { O-GDM } & \text { Offspring of women with GDM } \\ \text { O-T1DM } & \text { Offspring of women with type } 1 \text { diabetes }\end{array}$

\section{Introduction}

Gestational diabetes mellitus (GDM) is defined as glucose intolerance of varying severity and is present in around 2-6\% 
of all pregnancies in Europe, with regional variations [1]. GDM is therefore one of the most common pregnancy disorders. If left untreated, the risk of complications such as preeclampsia and preterm delivery is increased. GDM also results in excess growth of the fetus, causing problems during labour and delivery for both mother and offspring, including birth lacerations, delivery by Caesarean section and problems during the neonatal period [2]. Many of these complications can be prevented by proper treatment during pregnancy $[3,4]$. Furthermore, in most women with GDM, glucose tolerance normalises shortly after delivery [5]. Thus, following a pregnancy complicated by GDM, most women will experience normalised glucose tolerance a few days after birth, and the vast majority will have a healthy baby. Unfortunately, reality paints a less optimistic picture of the future, because GDM can have long-lasting health consequences for both mother and child.

\section{Mother}

The original diagnostic criteria for GDM by O'Sullivan and Mahan [6] were based on the maternal risk of developing diabetes later in life, and not on the perinatal risk. Thus, the knowledge that women with elevated glucose levels during pregnancy are at an increased risk of subsequent diabetes is not new. This has been shown in different populations with various risk estimates, depending on the populations studied and the GDM criteria used [7].

We have found interesting trends in our studies on the development of diabetes after GDM in pregnancy in Denmark over the years. In our first follow-up study, performed a median of 6 years after pregnancy, in 241 women with previous diettreated GDM between 1978 and 1985, we found that almost $18 \%$ had developed diabetes ( $4 \%$ type 1 diabetes and $14 \%$ type 2 diabetes) and 17\% had prediabetes (defined as impaired glucose tolerance and/or impaired fasting glucose) [8]. Some 13 years later we re-invited the same women for another follow-up study [9]. At this time, after a median of 19 years, $37 \%$ of the 151 participants had diabetes (5\% type 1 diabetes, $32 \%$ type 2 diabetes), while $29 \%$ had prediabetes, meaning only a third had normal glucose tolerance. Simultaneously, we investigated a cohort of 330 women who had diet-treated GDM in the period between 1987 and 1996. Among this newer cohort, followed for a median of 7 years, $41 \%$ had diabetes $(4 \%$ type 1 diabetes, $37 \%$ type 2 diabetes) and $26 \%$ had prediabetes [9]. Multivariate analysis revealed that the risk of developing diabetes in the new cohort was threefold that in the old one. The main difference between the two cohorts was that the prepregnancy BMI was three units $\left(\mathrm{kg} / \mathrm{m}^{2}\right)$ higher in the new cohort than in the older cohort. In accordance with other studies, we found that increasing pre-pregnancy BMI, higher fasting glucose at GDM diagnosis, early gestational age at GDM diagnosis and post-partum impaired glucose tolerance were risk factors for the subsequent development of overt diabetes. More than half of the women with type 2 diabetes were diagnosed during the course of the study, and were thus unaware of the fact that they had the disease prior to participation. Furthermore, it was found that the risk of the metabolic syndrome in women with previous GDM was over three times that in the general group, even when adjusted for age and BMI [10]. Thus, our Danish data are in accordance with other studies. A review by Bellamy et al [7] reported that in women with previous GDM the risk of developing diabetes is more than seven times that in women who had a normoglycaemic pregnancy, underlining the importance of prevention or postponement of development of diabetes and cardiovascular disease in these women. It has been estimated that GDM is the best known predictor of type 2 diabetes, and that approximately one-third of women with type 2 diabetes may have had previous GDM [11]. Thus, a large potential exists for preventing or delaying the onset of type 2 diabetes in these women. Epidemiological studies indicate that women with previous GDM who are physically active or do not gain weight after pregnancy have a reduced risk of progressing to overt diabetes $[12,13]$. In accordance with this, in randomised controlled trials, lifestyle intervention and medical treatment have both been shown to decrease the number who progress to diabetes by approximately $50 \%$ in women with previous GDM $[14,15]$.

Most guidelines recommend breastfeeding, a lifelong healthy lifestyle including weight loss if necessary, as well as an OGTT 2-6 months post-delivery. After delivery, glucose tolerance should be assessed every 1-3 years [16] using either fasting glucose, OGTT or $\mathrm{HbA}_{1 \mathrm{c}}$. In spite of these guidelines, systematic follow-up programmes facilitating early diagnosis and intervention are generally lacking in the routine clinical set-up for the majority of women with GDM worldwide, probably because of a combination of several reasons. When women with GDM are discharged from hospital after birth, determining who has responsibility for subsequent routine followup visits can be a difficult task - is it the patient herself, the general practitioner, the endocrinologist or the health authorities? While most women with GDM are highly motivated, capable of making the necessary lifestyle changes and adhering to a strict treatment regimen during pregnancy, things become more difficult when glucose tolerance returns to normal after delivery and daily life challenges pile up. These women are often mothers with full-time jobs, and in a previous study from Copenhagen few were able to maintain a healthy lifestyle [17]. Even in settings where the women are invited and the post-partum OGTT is offered free of charge, the attendance rate remains at approximately $50 \%$. It seems logical to make this lifelong post-partum care, control and guidance part of the public healthcare system, for example, in association with the routine child examinations and vaccinations, which most mothers will attend together with their offspring. 


\section{Offspring}

While the long-term maternal metabolic risk after GDM has long been well established, the long-term risks for the offspring are less well explored. Elegantly performed animal studies have demonstrated that offspring of mothers with GDM are at increased risk of GDM, diabetes, obesity, cardiovascular disease and structural hypothalamic changes $[18,19]$. Furthermore, these animal studies have documented that normalisation of maternal blood glucose levels during pregnancy prevents these negative outcomes. While studies in adult offspring are limited, several human studies exist in children from different populations, involving a mix of different maternal diabetes types [20-22]. The results of these studies are in line with the animal studies, the majority finding increased risk of diabetes and obesity in children exposed to maternal diabetes.

The following is an overview of our own follow-up studies of adult offspring of women with GDM. Offspring of women with GDM (O-GDM), type 1 diabetes (O-T1DM) and from the background population (O-BP) were followed until age 18-27 years $[23,24]$. Prediabetes/diabetes was present in $21 \%$ of O-GDM and $11 \%$ of O-T1DM compared with $4 \%$ of O-BP. When adjusted for confounders, the risk of prediabetes/diabetes in O-GDM was eight times that in O-BP. In O-T1DM, who have a different genetic background with respect to risk of type 2 diabetes, the risk of prediabetes/diabetes was four times that in $\mathrm{O}-\mathrm{BP}$, with a positive association with maternal glucose levels in late pregnancy. The risk of developing the metabolic syndrome in O-T1DM and O-GDM was two and four times that in O-BP, respectively, and in O-GDM the risk increased with increasing maternal glucose levels as assessed by OGTT during pregnancy [24]. Finally, the risk of being overweight during adulthood was two times that in O-BP for both O-GDM and O-T1DM. These findings are in line with a direct effect of maternal hyperglycaemia, in combination with other factors, on the risk of offspring developing diabetes, overweight and the metabolic syndrome. However, the specific underlying pathogenic mechanisms are unknown.

Based on an OGTT, we found that exposure to intrauterine hyperglycaemia was associated with impaired insulin sensitivity and relatively impaired insulin secretion in adulthood, both of which are characteristics of type 2 diabetes [25]. Chronic inflammation has been closely associated with type 2 diabetes and the metabolic syndrome, and we set out to investigate this as well. Levels of high-sensitivity CRP, IL-6 and YKL40 were investigated in O-GDM but no abnormalities were found [26], indicating that exposure to intrauterine hyperglycaemia does not affect the level of chronic inflammation in O-GDM. Finally, no major changes in incretin and glucagon function (during OGTT) were found in the offspring, but slightly reduced levels of glucagon-like peptide 1 in the fasting state, and reduced suppression of glucagon during OGTT may contribute to the increased risk of glucose intolerance among O-GDM [27].

In theory, this abnormal metabolic risk profile in O-GDM could be due to epigenetic changes induced by maternal hyperglycaemia, and a second follow-up of our cohort is currently underway, in which target tissues, such as skeletal muscle and subcutaneous adipose tissue, have been obtained for epigenetic analyses.

It has been discussed whether exposure to intrauterine hyperglycaemia can induce impaired cognitive function in the offspring. We found a slightly lower cognitive score in O-GDM in univariate analysis, but when adjusting for parental educational level and socioeconomic status this difference disappeared [28]. Other studies find varying results, and there is currently no solid evidence indicating that maternal GDM causes impaired cognitive function in the offspring [22].

A key question that remains to be answered is whether optimal treatment of maternal hyperglycaemia can affect the long-term prognosis of the offspring, as has been found in animal studies $[18,19]$. For this purpose, long-term followup studies of offspring of mothers from GDM treatment trials are required. Fortunately, two studies already exist [29, 30], with follow-up ranging from 4 to 10 years. Both studies used BMI as the primary outcome and found no differences in offspring BMI, regardless of whether maternal GDM was treated or untreated. In a sub-analysis of one of the studies [30], it was found that treatment of the mother was associated with increased insulin sensitivity, lower fasting glucose and a lower rate of impaired glucose tolerance in female offspring only. These findings are discouraging compared with the wellestablished effect of treatment on short-term perinatal outcome $[3,4]$. The apparent general lack of treatment effect on the long-term prognosis of the offspring could be due to (1) lack of statistical power, since the studies were not originally designed for long-term follow-up studies of offspring; (2) lack of follow-up time, since some studies indicate that the beneficial effect of treatment occurs at an older age [31]; or (3) insufficient maternal treatment with respect to the longterm effects on the offspring.

\section{Conclusion}

In conclusion, GDM can have a marked impact on the future health of both mother and offspring. The prevalence of GDM is increasing in most populations, and it is very likely that GDM plays a significant role in the global diabetes epidemic. Diabetes begets diabetes.

Effective intervention strategies for preventing or reducing metabolic derangement in the mother after delivery are available, but they need to be generally implemented in the clinical setting. Concerning prevention in the offspring, our knowledge is still too limited to recommend specific programmes. 
Duality of interest The authors declare that there is no duality of interest associated with this manuscript.

Contribution statement All authors were responsible for drafting the article and revising it critically for important intellectual content. All authors approved the version to be published.

\section{References}

1. Buckley BS, Harreiter J, Damm P et al (2012) Gestational diabetes mellitus in Europe: prevalence, current screening practice and barriers to screening. A review. Diabet Med 29:844-854

2. Metzger BE, Lowe LP, Dyer AR et al (2008) Hyperglycemia and adverse pregnancy outcomes. N Engl J Med 358:1991-2002

3. Crowther CA, Hiller JE, Moss JR, McPhee AJ, Jeffries WS, Robinson JS (2005) Effect of treatment of gestational diabetes mellitus on pregnancy outcomes. N Engl J Med 352:2477-2486

4. Landon MB, Spong CY, Thom E et al (2009) A multicenter, randomized trial of treatment for mild gestational diabetes. N Engl J Med 361:1339-1348

5. Damm P (2009) Future risk of diabetes in mother and child after gestational diabetes mellitus. Int J Gynaecol Obstet 104(Suppl 1): S25-S26

6. O'Sullivan JB, Mahan CM (1964) Criteria for the oral glucose tolerance test in pregnancy. Diabetes 13:278-285

7. Bellamy L, Casas JP, Hingorani AD, Williams D (2009) Type 2 diabetes mellitus after gestational diabetes: a systematic review and meta-analysis. Lancet 373:1773-1779

8. Damm P, Kuhl C, Bertelsen A, Molsted-Pedersen L (1992) Predictive factors for the development of diabetes in women with previous gestational diabetes mellitus. Am J Obstet Gynecol 167:607-616

9. Lauenborg J, Hansen T, Jensen DM et al (2004) Increasing incidence of diabetes after gestational diabetes: a long-term follow-up in a Danish population. Diabetes Care 27:1194-1199

10. Lauenborg J, Mathiesen E, Hansen T et al (2005) The prevalence of the metabolic syndrome in a Danish population of women with previous gestational diabetes mellitus is three-fold higher than in the general population. J Clin Endocrinol Metab 90:4004-4010

11. Cheung NW, Byth K (2003) Population health significance of gestational diabetes. Diabetes Care 26:2005-2009

12. Bao W, Tobias DK, Bowers K et al (2014) Physical activity and sedentary behaviors associated with risk of progression from gestational diabetes mellitus to type 2 diabetes mellitus: a prospective cohort study. JAMA Intern Med 174:1047-1055

13. Bao W, Yeung E, Tobias DK et al (2015) Long-term risk of type 2 diabetes mellitus in relation to BMI and weight change among women with a history of gestational diabetes mellitus: a prospective cohort study. Diabetologia 58:1212-1219

14. Ratner RE, Christophi CA, Metzger BE et al (2008) Prevention of diabetes in women with a history of gestational diabetes: effects of metformin and lifestyle interventions. J Clin Endocrinol Metab 93: $4774-4779$
15. Buchanan TA, Xiang AH, Peters RK et al (2002) Preservation of pancreatic beta-cell function and prevention of type 2 diabetes by pharmacological treatment of insulin resistance in high-risk hispanic women. Diabetes 51:2796-2803

16. American Diabetes Association (2015) Management of diabetes in pregnancy. Diabetes Care 38:S78-S79

17. Stage E, Ronneby H, Damm P (2004) Lifestyle change after gestational diabetes. Diabetes Res Clin Pract 63:67-72

18. Harder T, Aerts L, Franke K, Van Bree R, Van Assche FA, Plagemann A (2001) Pancreatic islet transplantation in diabetic pregnant rats prevents acquired malformation of the ventromedial hypothalamic nucleus in their offspring. Neurosci Lett 299:85-88

19. Aerts L, Van Assche FA (2006) Animal evidence for the transgenerational development of diabetes mellitus. Int J Biochem Cell Biol 38:894-903

20. Silverman BL, Rizzo T, Green OC et al (1991) Long-term prospective evaluation of offspring of diabetic mothers. Diabetes 40(Suppl 2):121-125

21. Dabelea D, Hanson RL, Lindsay RS et al (2000) Intrauterine exposure to diabetes conveys risks for type 2 diabetes and obesity: a study of discordant sibships. Diabetes 49:2208-2211

22. Fraser A, Lawlor DA (2014) Long-term health outcomes in offspring born to women with diabetes in pregnancy. Curr Diab Rep $14: 489$

23. Clausen TD, Mathiesen ER, Hansen T et al (2008) High prevalence of type 2 diabetes and pre-diabetes in adult offspring of women with gestational diabetes mellitus or type 1 diabetes: the role of intrauterine hyperglycemia. Diabetes Care 31:340-346

24. Clausen TD, Mathiesen ER, Hansen T et al (2009) Overweight and the metabolic syndrome in adult offspring of women with diettreated gestational diabetes mellitus or type 1 diabetes. J Clin Endocrinol Metab 94:2464-2470

25. Kelstrup L, Damm P, Mathiesen ER et al (2013) Insulin resistance and impaired pancreatic beta-cell function in adult offspring of women with diabetes in pregnancy. J Clin Endocrinol Metab 98: 3793-3801

26. Kelstrup L, Clausen TD, Mathiesen ER, Hansen T, Damm P (2012) Low-grade inflammation in young adults exposed to intrauterine hyperglycemia. Diabetes Res Clin Pract 97:322-330

27. Kelstrup L, Clausen TD, Mathiesen ER, Hansen T, Holst JJ, Damm P (2015) Incretin and glucagon levels in adult offspring exposed to maternal diabetes in pregnancy. J Clin Endocrinol Metab 100: 1967-1975

28. Clausen TD, Mortensen EL, Schmidt L et al (2013) Cognitive function in adult offspring of women with gestational diabetesthe role of glucose and other factors. PLoS One 8:e67107

29. Gillman MW, Oakey H, Baghurst PA, Volkmer RE, Robinson JS, Crowther CA (2010) Effect of treatment of gestational diabetes mellitus on obesity in the next generation. Diabetes Care 33:964968

30. Landon MB, Rice MM, Varner MW et al (2015) Mild gestational diabetes mellitus and long-term child health. Diabetes Care 38:445452

31. Crume TL, Ogden L, Daniels S, Hamman RF, Norris JM, Dabelea $\mathrm{D}$ (2011) The impact of in utero exposure to diabetes on childhood body mass index growth trajectories: the EPOCH study. J Pediatr 158:941-946 